\title{
El desarrollo de sistemas ERP: elementos para un enfoque por procesos
}

\author{
MAssimo LozZI \\ Prenatal \\ Piercarlo Maggiolini \\ Politecnico di Milano \\ E-mail: piercarlo.maggiolini@polimi.it \\ Ramon Salvador Vallès \\ Univerdidad Politecnica de Catalunya
}

\section{Resumo}

Este artigo oferece uma contribuição para uma revisão das metodologías, e do horizonte teórico que tradicionalmente caracteriza a "Engenharia da informaçao". O artigo define as opções epistemológicas sobre as quais, se deve fundamentar uma metodología de desenvolvimento de projetos de sistemas da informação, e se individualizam as possíveis orientações estratégicas que caracterizam o desenvolvimento de projetos.

A finalidade que se persegue é: 1] desenvolver modelos interpretativos e de suporte ao desenvolvimento de projetos, que permitam superar as simplificações próprias dos enfoques BRP dentro de uma visão comum, de acordo com os processos; 2] melhorar a compreensão das relações recíprocas entre os processos de desenvolvimento de projetos e os "processos projetados" assumindo, como ponto de partida, a natureza organizativa comum; 3) reforçar teórica e metodologicamente o desenvolvimento de sistemas da informação e dos sistemas ERP em particular.

\section{Palavras-chave}

Engenharia da informação, Planejamento de pesquisa empresarial, Enfoque por processos, Processo de reengenharia de negócios.

\section{The development of ERP systems: elements for a process-based approach.}

\begin{abstract}
This paper contributes to a re-examination of the methodologies and theoretical horizon that has traditionally characterised information engineering. The paper defines the epistemological choices that are the foundation for information system project development and individualises the possible strategic orientations that characterise project development.

The objectives pursued are: 11 to develop interpretative models and support for project development with a processbased approach in order to overcome the simplifications inherent to BPR; 2J improve our understanding of the reciprocal relationships between project development processes and "processes projected", in the hypothesis of a common organizational nature; and 3 J reinforce the development of information systems and particularly ERP from a theoretical and methodological standpoint.
\end{abstract}

Key words

Information Engineering, Enterprise Resource Planning, Process-based approach, Business Process Reengineering. 


\section{INTRODUCCIÓN}

Ciertamente el debate sobre las relaciones entre Information and Communication Technology (ICT) y la organización no es nuevo, pero ahora debe confrontarse con dos novedades relevantes:

- la emergencia del concepto de proceso como referencia fundamental del desarrollo organizativo;

- el desarrollo extraordinario del potencial de aplicación de la ICT al punto de hacer casi prácticamente indistinguibles las actividades de desarrollo organizativo y desarrollo de sistemas de información.

La figura tradicional del analista de procedimientos dirigida a identificar y describir los procedimientos organizativos automatizables pierde progresivamente significado. Adquiere cada vez más importancia la figura del analista de proceso "llamado a participar activamente en la fase de diagnosis y rediseño de los procesos, identificando los aspectos críticos y las tipologías de las actividades, ilustrando las posibilidades ofrecidas a las modernas TI y evaluando la conveniencia del cambio propuesto" (BRACCHI Y MOTTA, 1998, pág. 58). El desarrollo de los sistemas de información sitúa, por ello, en primer lugar, una investigación activa de las áreas de congruencia entre la complejidad que caracteriza el ambiente de la organización, la elección y las posibilidades técnicas, las elecciones de la estructuración de los procesos (MIGLIARESE, FERIOLI, 1996).

Frente a tal evolución, las metodologías tradicionales de desarrollo de sistemas de información, centradas en la figura del analista del procedimiento, resultan siempre más inadecuadas para soportar el papel mucho más complejo del analista de proceso.

Sin este cambio de perspectiva, el énfasis sobre el potencial del cambio asociado a la ICT corre el riesgo de hacerse un mito más que una realidad efectiva. Las experiencias hechas con los siempre más difusos sistemas ERP (Enterprise Resource Planning), por ejemplo, aunque siempre en continua evolución, muestran como, para adoptar un estándar de hecho en el campo de los sistemas de información, se introducen y se generan nuevas rigideces (RAVAGNANI, 2000a, 2000b).

Las mismas insuficiencias teóricas y metodológicas de las diversas técnicas BPR (Business Process Reengineering), que acompañan la implantación de los nuevos sistemas integrados, mirando de conseguir en el más breve tiempo posible mejoras "drásticas" de la performance de la empresa no son probablemente extrañas a todo esto.

En este marco, el objetivo del presente trabajo es el de proveer una contribución para una revisión (un cambio de opinión) de las metodologías y del horizonte teórico que tradicionalmente ha caracterizado la information enginee- ring. Tal operación requiere, según nuestra opinión, una estrecha comparación con las corrientes del pensamiento organizativo epistemológicamente compatible con una visión según los procesos de la organización con el fin de:

- desarrollar algunos modelos interpretativos y de soporte al desarrollo de proyectos que permitan superar las simplificaciones propias de los enfoques BPR (MIGLIARESE, FERIOLI, IAZZOLINO, 1999; PEPPARD, 1994; COOMBS, HULL, 1997) dentro de una visión común según procesos;

- mejorar la comprensión de las relaciones recíprocas entre los procesos de desarrollo de proyectos y los "procesos proyectados" asumiendo, como dato de partida, la naturaleza organizativa común;

- reforzar teórica y metodológicamente el desarrollo de sistemas de información y de los sistemas ERP en particular.

\section{El desarrollo de sistemas de información como proceso organizativo}

El punto de partida de nuestra exposición es la concepción del proceso de desarrollo de proyectos en términos de un proceso organizativo.

El horizonte teórico y epistemológico dentro del cual nos moveremos, relativo al concepto de proceso organizativo, es aquel de la Acción Organizativa (MAGGI, 1990; MAGGI, ALBANO, 1997; MASINO, 1997, 2000a). Los motivos de fondo que nos conducen a ésta elección son:

- la orientación según procesos que caracteriza el acercamiento próximo a la corriente más innovativa tanto del ámbito de la investigación sociológica como de la práctica de dirección y de consultoría $(\mathrm{BPR})^{1}$;

- la base teórica sobre la que se basa, radica en las corrientes del pensamiento fuertemente reconocido y consolidado (en particular Simon y Thompson) y que desde siempre han constituido un puente entre la teoría de la organización y la teoría de los sistemas de información (se piensa en los conceptos de racionalidad limitada y de interdependencia);

- la caracterización de los procesos organizativos en términos de flujos de acciones y decisiones, muy próxima al concepto de flujo informativo que caracteriza el «dominio lingüístico» de los desarrolladores de proyectos informáticos.

Dentro de esta línea interpretativa los procesos organizativos se conciben como procesos de acciones y decisiones orientadas a una finalidad según una racionalidad intencional y limitada (MAGGI 1990; MAGGI, ALBANO, 1997).

Esta concepción aparece suficientemente general para comprender una amplia clase de procesos entre los cuales también hay los procesos de desarrollo de proyectos. 
Estos últimos, también en el ámbito intuitivo, son en efecto caracterizados como:

- procesos de acciones y decisiones;

- orientados a un objetivo (el desarrollo de un proyecto de un SI);

- caracterizados desde la racionalidad intencional y limitados donde el resultado del proceso (el SI) es difícilmente interpretable como la mejor de las soluciones posibles, pero es sin embargo el fruto de la valoración orientada por el criterio de satisfacción. Los resultados del proceso son, para usar el lenguaje de la Investigación Operativa, fruto de elecciones y de procedimientos heurísticos que solo ocasionalmente conducen a soluciones óptimas.

Refiriéndonos siempre a los elementos fundamentales de la aproximación teórica que han seguido (MAGGI, 1990; MAGGI, ALBANO, 1997; MASINO, 1997), el proceso de desarrollo de proyectos pudo ser representado como un proceso decisional en el que son analíticamente identificables:

- decisiones estratégicas, relativas a Ravagni (2000):

- campo de acción del proyecto en términos de amplitud, radicalidad, grado de riesgo, prioridad, software y hardware elegidos para la implantación, servicios requeridos, etc.

- recursos necesarios: financieros, competencia interna y externa;
- estructura institucional de soporte del proyecto (órganos de gobierno).

- decisiones técnicas, relativas a las acciones de transformación del input (el SI precedente) en output (el nuevo SI),

- decisiones de coordinación y control de la actividad, relativas a la modalidad de coordinación y control de las acciones de transformación del SI (acciones técnicas) en términos de criterios de paralelización, secuencialización y retroacción entre las actividades;

- decisiones de coordinación y control de la persona, relativas a las atribuciones (quien hace cada cosa), a las reglas y procedimientos del desarrollo de la actividad, a los criterios de gestión de los cambios informativos entre las unidades comprometidas en el proyecto.

Se hace referencia a la Figura 1.

Los rectángulos indican los momentos decisionales en los que es descompuesto analíticamente el proceso. La flecha llena indica relaciones de precedencia lógica mientras la flecha a trazos relaciones de retroalimentación. Esto para dar cuenta de las relaciones de congruencia y no de determinación entre los diversos componentes. Son entonces siempre posibles, y necesarias, relaciones de alineamiento recíproco entre las elecciones que caracterizan los momentos decisionales. Cada momento decisional define las premisas a las deci-

Figura 1: El proceso de desarrollo de proyectos: un modelo basado en la teoría de la acción organizativa.

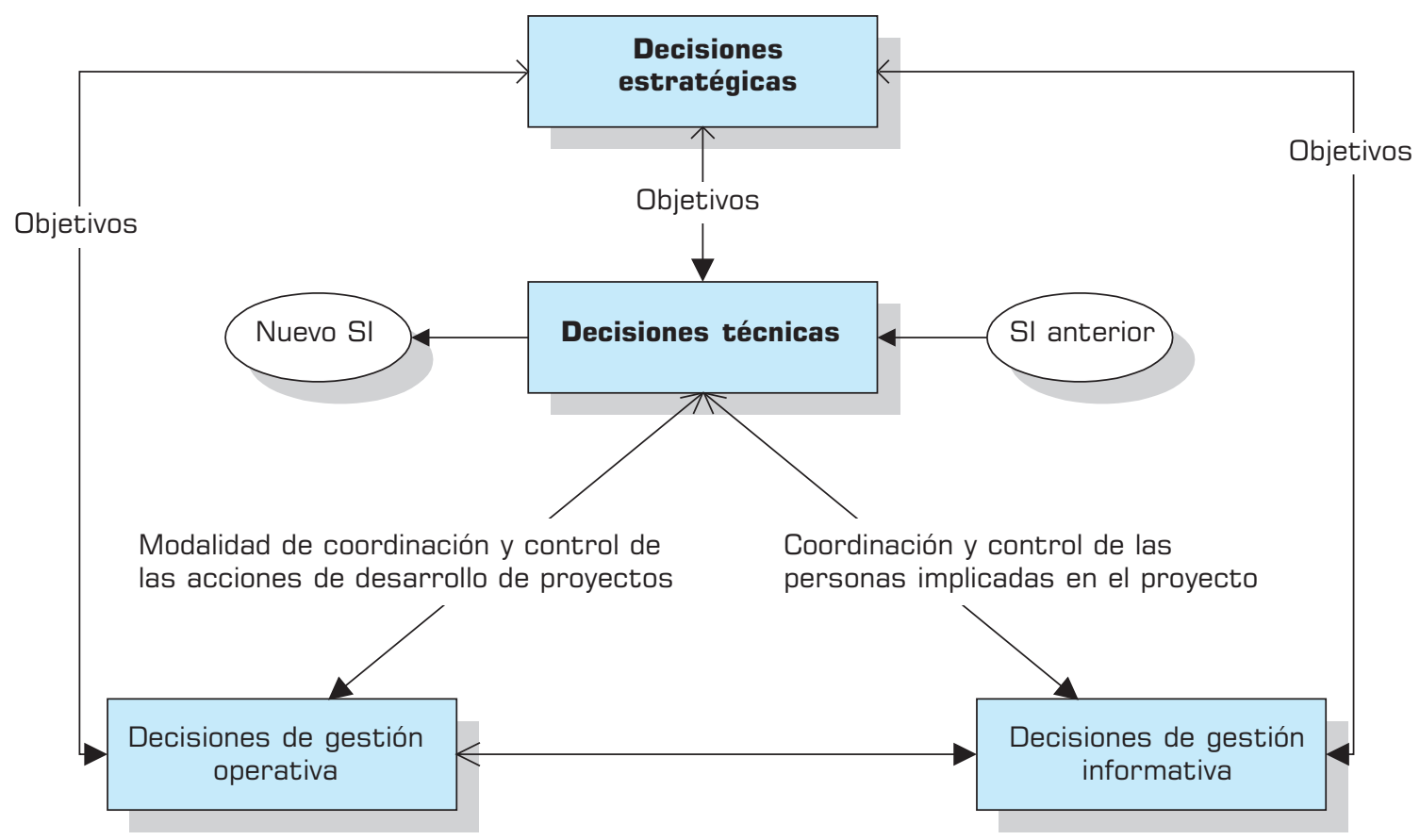


siones de los momentos sucesivos (SIMON, 1947) es decir el conjunto de los vínculos y contingencias que no condicionan las elecciones (THOMPSON, 1967).

\section{El sistema de información como proceso organizativo}

En una teoría de la organización exhaustiva la comunicación debería ocupar un lugar central, ya que la estructura, la extensión, las dimensiones de una organización son casi totalmente determinadas por técnicas de comunicación. (BARNARD, 1938; trad. it. 1970)

Las estructuras organizativas con sus diversas dimensiones, sofisticación tecnológica, grado de complejidad y formalización son planificadas para ser o para volverse sistemas para el tratamiento de la información. (HALL, 1977)

El sistema de las comunicaciones es la organización. La comunicación organizativa es la teoría de la organización del futuro. (PACE, 1983)

Las expresiones citadas en la portada inicial permiten dar luz a la estrecha relación existente entre los conceptos de proceso organizativo y sistema de información. Ellas expresan la necesidad de superar una visión estática de los sistemas de información, centrada sobre la tecnología, hacia una interpretación que consienta tomar los informes recíprocos entre procesos de transformación de las informaciones y procesos organizativos. Eso requiere un trabajo de recomposición de las referencias conceptuales propias del análisis de los sistemas de información dentro del cuadro interpretativo delineado por las principales corrientes de la teoría de la organización. En particular, respecto a las finalidades delineadas, nos parece bastante ardiente la comparación con la línea teórica que lleva a cabo a Simon y Thompson.

Desde nuestro punto de vista, el SI, al igual que el proceso de desarrollo de proyectos, puede ser interpretado como un proceso organizativo.
Entenderemos por lo tanto, en una primera aproximación, el SI como un proceso de acciones y decisiones orientado a una finalidad por medio de técnicas de transformación de las informaciones.

Vamos a intentar ahora de caracterizar mejor los componentes analíticos del proceso.

El proceso de acciones y decisiones, sobre el plano analítico, se puede descomponer en ${ }^{2}$ (Figura 2):

- Flujos estratégicos: relativos a las relaciones entre los objetivos que caracterizan el proceso (se ve en la Tabla 1)

- Flujos de transformación: relativos a las acciones de transformación del input en output necesarias para satisfacer los objetivos.

- Flujos de gestión: relativos a la modalidad de gestión de las relaciones entre las acciones técnicas (flujos de gestión operativa) y la modalidad de gestión de los intercambios de información entre los actores comprometidos en el desarrollo (flujos de gestión informativa). Estos expresan la componente estructural del proceso en términos de reglas de coordinación y control de las actividades y de las personas (MAGGI, 1990).

Intentemos ahora aclarar mejor los conceptos arriba expuestos con un ejemplo. En ese sentido nos referiremos a un caso muy conocido en la literatura sobre gestión y que tiene que ver con la modalidad con que Hammer (1993) ha "reingenierizado" la contabilidad de los proveedores de la Ford $^{3}$. Utilizaremos el ejemplo en todo el resto del texto. En este párrafo nos limitaremos a una descripción as-is es decir relativa al proceso antes del reengineering. El ejemplo no pretende ser una descripción exhaustiva del proceso sino una descripción limitada a los aspectos necesarios para aclarar todo lo dicho hasta ahora.

Figura 2: Los procesos organizativos: caracterización en términos de flujos.

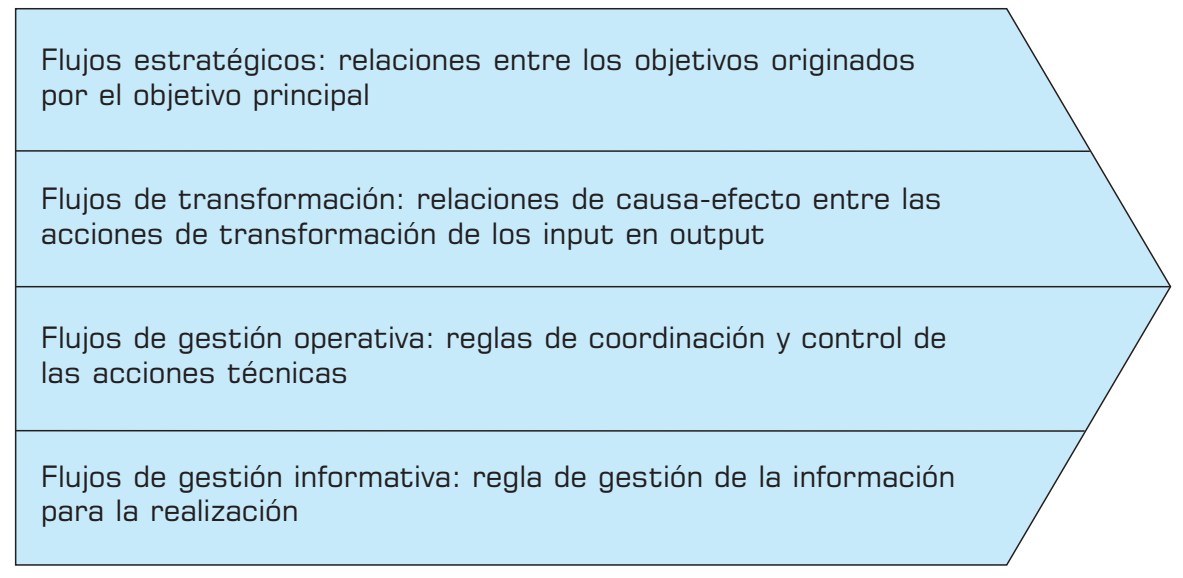


Empecemos con el análisis de los flujos estratégicos. Considérense las Figuras 3 y 4. Éstas describen respectivamente las interacciones de la contabilidad de los proveedores con procesos externos y los objetivos del proceso relativos a estas relaciones. Emerge claramente cómo la mission del proceso trata el control sobre todo el proceso de gestión de la orden de compra (OC) hasta el pago del proveedor que puede ocurrir sólo si AEM (albarán entrada mercancías), OC y factura son congruentes.

Consideremos ahora la Figura 5, en ella está indicado el flujo de transformación del proceso. Los elementos analíticos relevantes a este nivel son (MAGGI, 1990) las acciones de transformación (óvalos); los input y los output de las acciones de transformación; el proceso de transformación en términos de relación técnica entre las acciones de transformación (paralelismo, secuencia, feedback). En el caso en examen las relaciones son típicamente secuenciales con elevada separa- ción entre acciones operativas y acciones de control (la comprobación de congruencia de los documentos).

En la Figura 6 reflejamos los flujos de gestión operativa. En ella están indicadas las reglas de gestión de las relaciones entre las actividades. En la figura están indicadas con hexágonos relacionados con operadores lógicos (en el ejemplo solo AND). Así, por ejemplo, el cierre de la actividad de emisión de la orden de compra implica tanto el envío de la OC para la ejecución de la orden de parte de los proveedores, como el envío de una copia de la OC para juntar los documentos. Esto último puede ser activado sólo si todos los tres eventos indicados se han verificado. Nótese que se trata sobretodo de reglas de conexión dedicadas a especificar la modalidad de secuencialización de las actividades. No están en cambio esplicitadas reglas de retroacción entre las actividades. Por ejemplo no son esplicitadas reglas de retroacción en el caso de incongruencia de los documentos.

\section{Tabla 1: Objetivos y flujos estratégicos: profundizaciones.}

Asumiremos aquí la interpretación del concepto de objetivo propuesta por Thompson (1967). Antes que nada, Thompson distingue entre objetivos del proceso y criterios de valoración del proceso en cuanto a la persecución de los propios objetivos. Esta puntualización es importante porque los dos aspectos son frecuentemente confusos. Thompson (1967, p. 243 trad. it.) define los objetivos como "[...] los campos de acción futuros proyectados por aquellos que se encuentran en la coalición dominante.".

En este sentido, el concepto de objetivo es muy próximo al concepto de misión del proceso es decir del conjunto de los productos/servicios ofrecidos para satisfacer la exigencia de determinadas poblaciones. La misión del proceso tiene que entenderse sin embargo en sentido dinámico y político como punto de encuentro entre las posiciones que constituyen la coalición dominante o sea el conjunto de actores (externos o internos al proceso) respecto a los cuales el proceso es de alguna forma dependiente (por ejemplo clientes, proveedores, competidores, entes de reglamentación, etc. J. Vale la pena citar por completo algunos pasajes de Thompson (1967, pp. 242-243 trad. it.):

“Existe un peligro evidente en concretar la abstracción 'organización' suponiendo que ésta, la abstracción, tenga objetivos o deseos. Obtenemos bien poco, de todos modos, si nos trasladamos al otro extremo, sosteniendo que los objetivos de una organización son, en cierto modo, el conjunto de los objetivos de sus miembros individuales. (...) La concepción de los objetivos organizativos que hemos presentado aquí supera ambos problemas tradicionales: no hemos concretado la organización, ni hemos simplemente sumado las preferencias de todos sus miembros. En esta concepción, los objetivos organizativos son establecidos por los individuos; se trata sin embargo de individuos interdependientes, los cuales, colectivamente, tienen un control suficiente de los recursos organizativos para emplearlos en ciertas direcciones y apartarlos de otras."

En este sentido la mission del proceso se define en relación al task environment, con este término Thomson (1967, p. 102 trad. It.J se refiere al conjunto de los "sectores del ambiente que resultan relevantes o potencialmente relevantes para la definición del objetivo y para su consecución.".

Con lo cual, en conclusión, la mission del proceso es el resultado, dinámico, del consenso de los miembros del task environment sobre el campo de acción del proceso. Vale la pena citar otra vez a Thomson (p. 104 trad. It.):

"Utilizando el concepto de consenso sobre el campo, no tenemos necesidad de asumir que la afirmación formal de los objetivos reflejada en documentos, estatutos de constitución o declaraciones institucionales represente -de hecho- el criterio en base al cual viene juzgada la racionalidad y vienen realizadas las elecciones de las alternativas de acción. Ni hay necesidad de aceptar aquellas ideologías que insisten en considerar el beneficio como objetivo de la empresa."

El análisis de los flujos estratégicos estará entonces dirigido a identificar las relaciones entre el proceso y los elementos de su ambiente en términos estratégicos, es decir de expectativas sobre aquello que el proceso hará (mission del proceso]. Unido a este aspecto pero lógicamente posterior es el análisis de los indicadores de prestaciones utilizados para valorar el proceso en la persecución de los objetivos. 
Finalmente, en la Figura 7 se indica el flujo de gestión informativa. Ésa muestra las modalidades de desarrollo de las actividades (MAGGI, 1990). Los elementos analíticos son entonces las atribuciones de las tareas (quién hace qué), los instrumentos utilizados para el desarrollo de las tareas, los intercambios de información entre las unidades.

En el ejemplo, el símbolo del operario con la máquina de escribir indica una actividad poco informatizada. Están en cambio evidenciados intercambios elevados de información de adaptación recíproca entre las unidades debidos a las frecuentes incongruencias entre los documentos a juntar.

\section{Proceso organizativo, sistema de información e instrumentos informáticos}

Queremos ahora desarrollar algunas consideraciones sobre posibles recorridos interpretativos de las relaciones entre sistema de información, organización e instrumentos informáticos. No es nuestra intención dar cuenta de forma exhaustiva de este debate. Nos limitaremos aquí a indicar, en términos generales, algunas orientaciones posibles para poder situar, respecto a ellos la discusión que sigue.

Una primera orientación posible, y por otra parte bastante difusa, consiste en identificar, sustancialmente, el sistema de información con los instrumentos informáticos. Des-

Figuras 3 y 4: Contabilidad de proveedores: flujos estratégicos y objetivos.

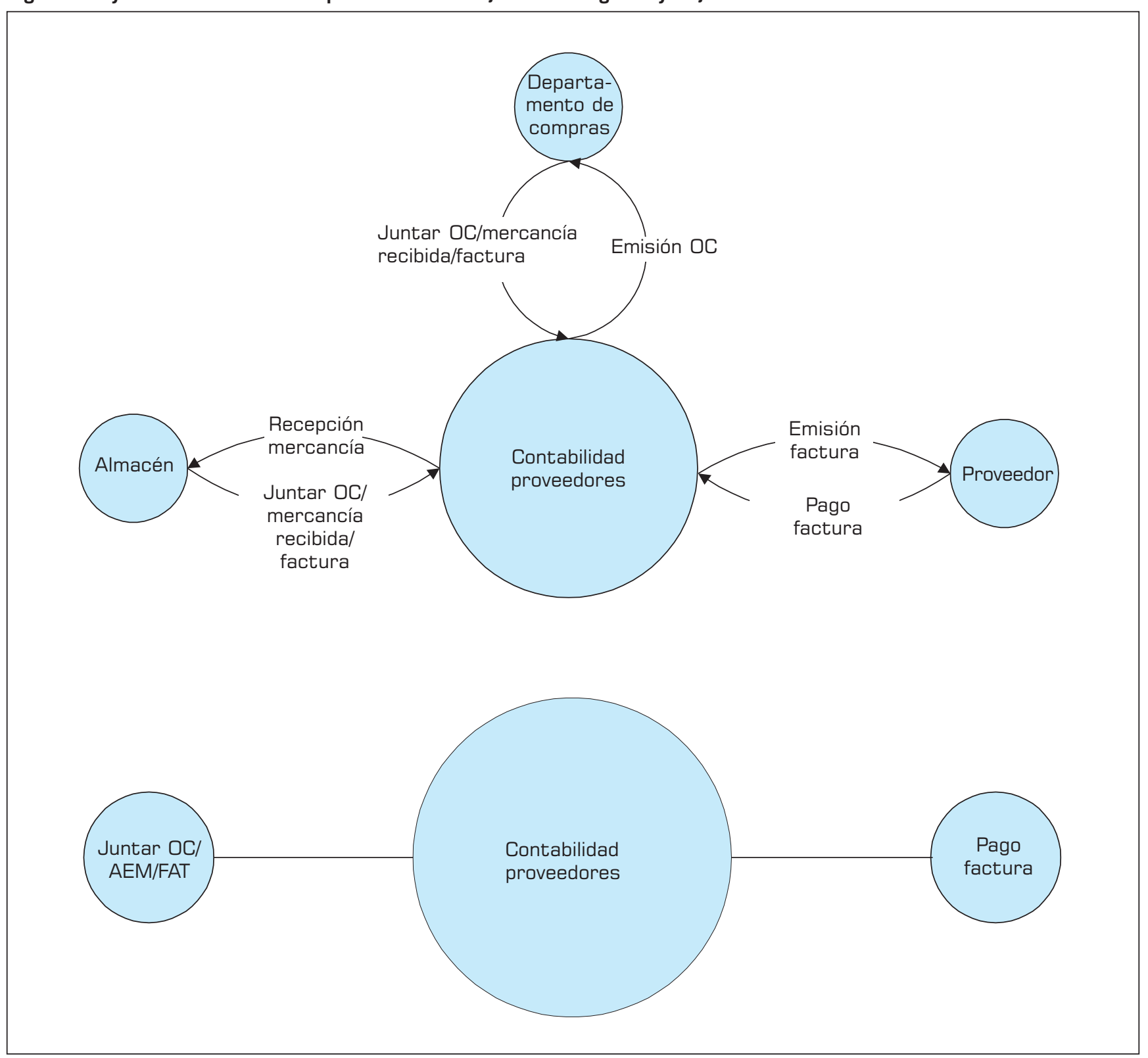


de esta óptica, el sistema de información y la organización son entidades separadas aunque en relación entre ellas. Es precisamente en referencia a la naturaleza de esta relación que se pueden individuar tres lógicas diferentes. (PONTIGGIA, 1997; MASINO, 1997; RAVAGNANI, 2000b):

1) Determinismo tecnológico: desde esta óptica la organización varía, o debe variar, de la forma determinada por las tecnologías empleadas. El determinismo puede ser de tipo "optimista", por ejemplo en términos de upskilling de los actores, descentralización del poder y de las decisiones, reducción de los niveles jerárquicos. Otra interpretación posible es sin embargo de tipo "pesimista", simétricamente, en términos de deskilling, centralización de las decisiones y aumento del control sobre los actores. Tanto la visión pesimista como la optimista son juntadas desde una orientación epistemológica de tipo positivista, de causaefecto one-way entre instrumentos técnicos y organización. Masino (1997) ha mostrado como los numerosos estudios empíricos de soporte de la tesis pesimista o de la optimista han llevado a resultados contradictorios, que han llevado a poner en discusión el paradigma positivista $\mathrm{y}$ determinista que orienta tales enfoques.

\section{Figura 5: Flujo de transformación.}

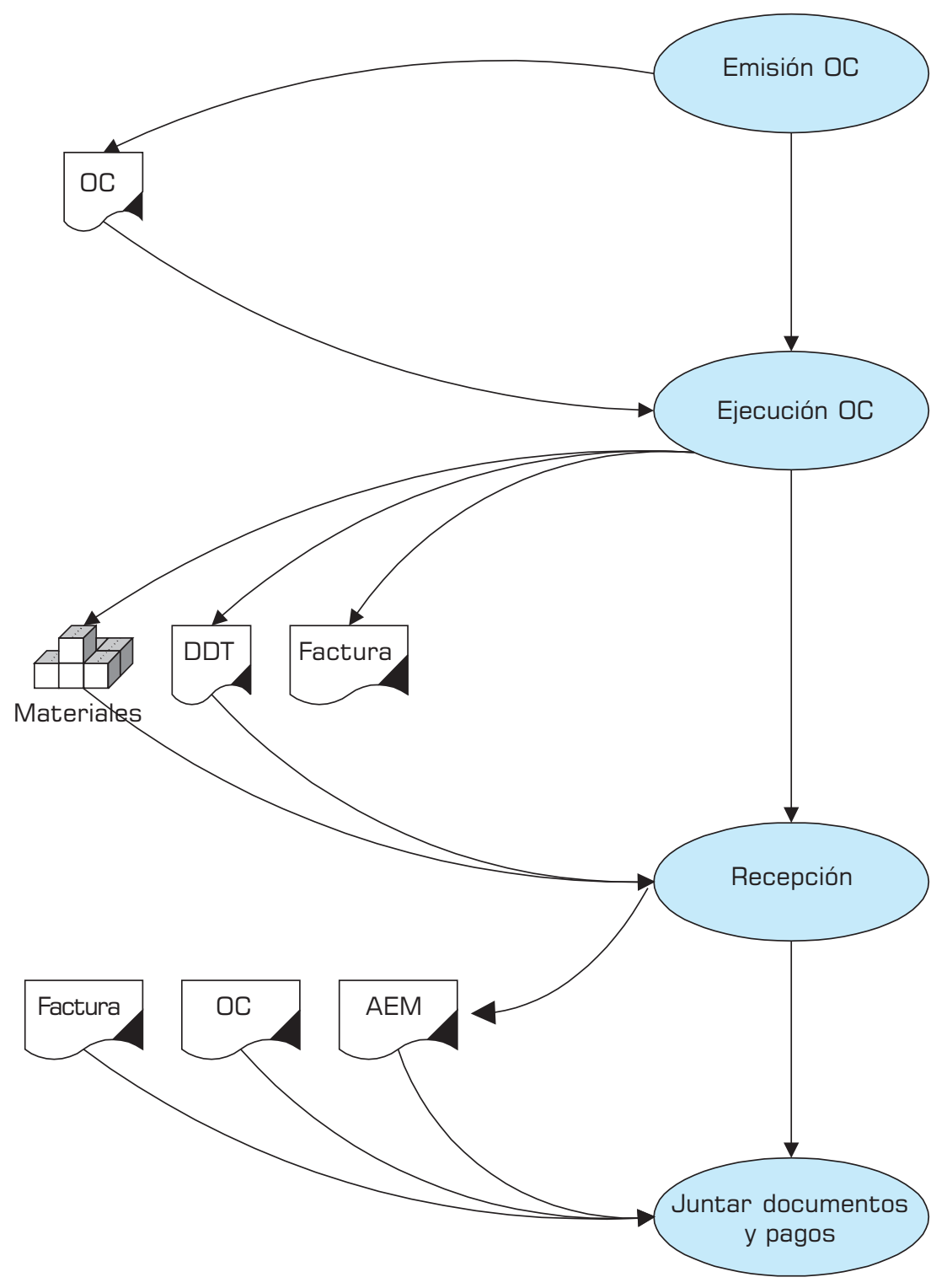


Figura 6: Flujos de gestión operativa.

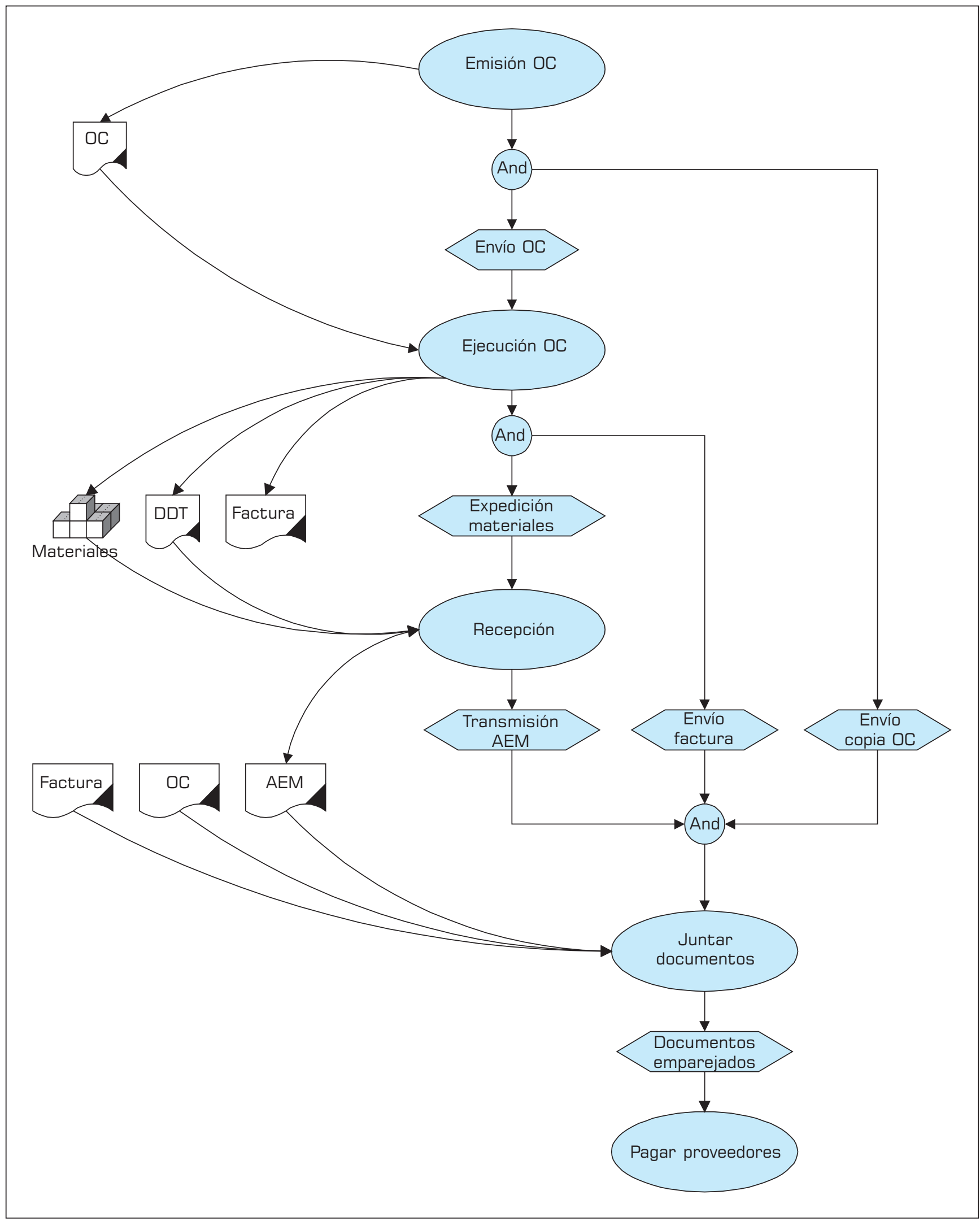


2) Determinismo organizativo: desde esta óptica la relación entre el sistema de información y la organización es aún determinista pero en la dirección opuesta. En otros términos, las características de los instrumentos que constituyen el sistema de información son determinadas completamente desde los requerimientos organizativos. Desde esta óptica, por lo tanto, el sistema de información, entendido como un conjunto de instrumentos de base

Figura 7: Flujo de gestión informativa.

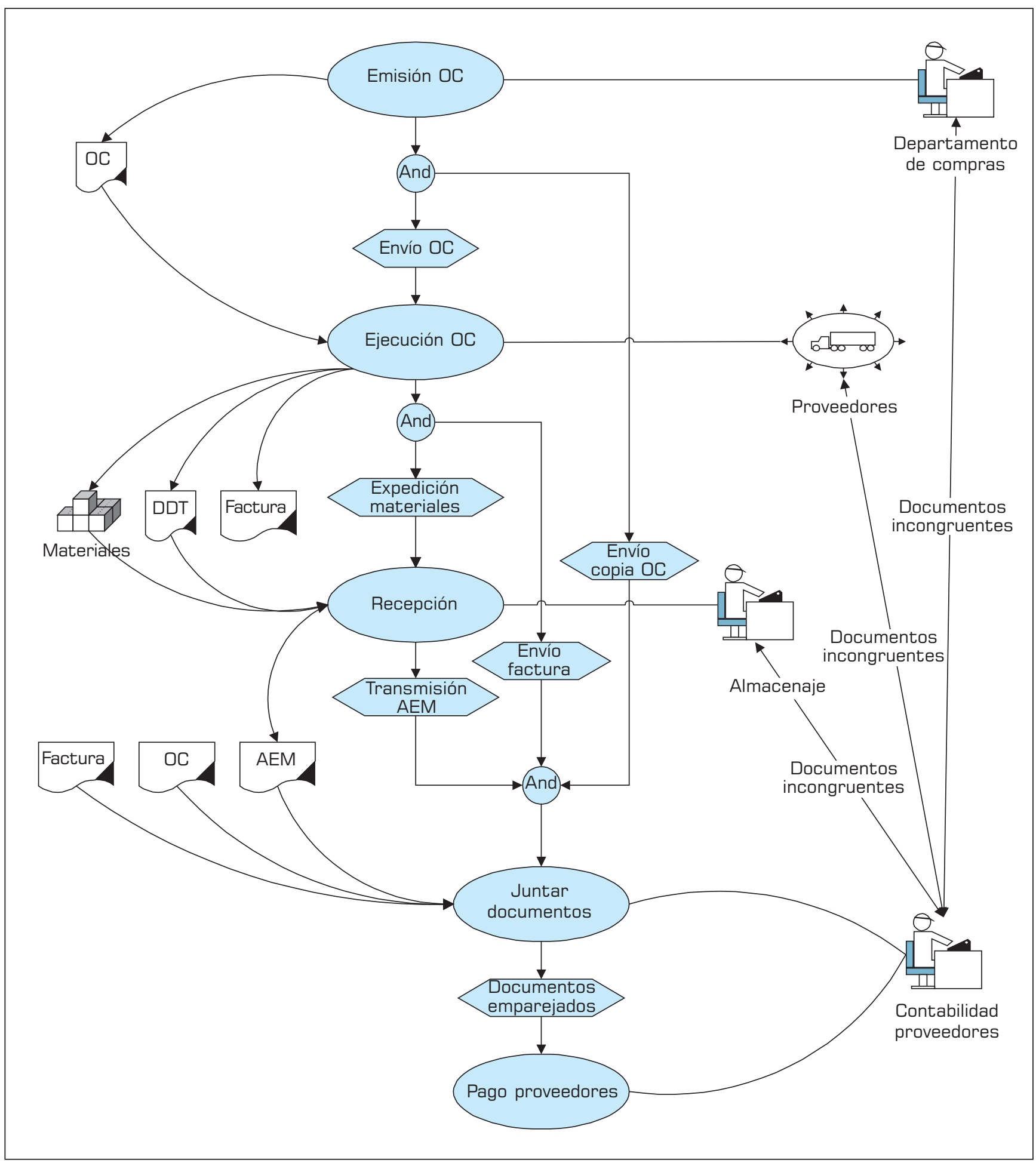


informática, es completamente determinado por los objetivos de la organización. Esta orientación, bastante difusa en la práctica de gestión y de consultoría, ha estado radicalmente puesta en crisis de los enfoques orientados al BPR (HAMMER y CHAMPY, 1993; DAVENPORT, 1994) que han mostrado como válida aun la relación inversa es decir como los objetivos y las disposiciones organizativas pueden ser radicalmente modificadas por las oportunidades ofrecidas por la nueva tecnología.

3) Lógica dual: esta línea interpretativa, relativamente reciente, hace referencia a la Structuration Theory de Giddens y encuentra en Orlikowski (1991, 1992), Tyre (1994), Barley (1986, 1990), De Sanctis y Poole (1994) sus exponentes más representativos. Desde esta óptica, la relación entre organización e instrumentos técnicos es bidireccional. Coherentemente con esta línea, el sistema de información encuentra en la disposición organizativa vínculos y fuerzas que posiblemente plasman sus características pero, análogamente, mediante el uso que de esto hacen los actores se pone un instrumento de cambio de las disposiciones organizativas. Este enfoque, indudablemente más adherente a la realidad de lo que no han sido los precedentes, tiene, sin embargo, un límite importante en la identificación del sistema de información con los instrumentos técnicos empleados (MASINO, 1997; 2000). Eso conduce a una separación entre sistema de información y el proceso organizativo que se traducen en dos entidades separadas pero en relación recíproca. Aunque las relaciones entre los dos son profundamente analizadas, la organización permanece en el fondo como conjunto vago e indistinto de estructura de legitimación, poder, significado. Esto hace esta orientación poco utilizable en términos de proyectos.

La óptica según la que colocaremos nuestra exposición es en cambio de tipo por procesos (MAGGI, 1990; MASINO, 1997, 2000). Desde esta óptica, no existe separación entre instrumentos tecnológicos y proceso. Ellos son una componente del proceso distinguible analíticamente pero no en su desarrollo concreto. En estos términos, las elecciones de los instrumentos no son separables de las elecciones que caracterizan los otros planos interpretativos (estratégicos, técnicos, estructurales). Sin embargo es distinta la interpretación de las elecciones de regulación del desarrollo de las actividades, de las cuales los instrumentos técnicos son una componente relevante, de la interpretación de las elecciones técnicas que atañen en cambio las relaciones de causaefecto entre acciones de transformación del input en output y que son ampliamente independientes de los instrumentos utilizados ${ }^{4}$. La distinción entre técnica e instrumentos técnicos es muy clara para quien se ocupa del desarrollo de proyectos de SI. Las fases iniciales de especificación funcional, en términos de relaciones input-transformación-output, deben ser, y esto forma parte "del $\mathrm{ABC}$ " del desarrollo de proyectos, absolutamente independientes de aquellas que serán los instrumentos utilizados. De forma diferente, las fases finales del proyecto, llevarán a la definición de las reglas de desarrollo de las actividades relativas a los instrumentos utilizados.

Desde la óptica por procesos, no existe predeterminación de una componente sobre otras, más bien influencia recíproca. Las elecciones estratégicas vinculan las elecciones técnicas y lo mismo ocurre para estas últimas respecto a las elecciones estructurales, pero cada una de las componentes indicadas provoca retroacción sobre las elecciones precedentes activando así una especie de "movimiento armónico" en el que es difícil distinguir el final y el inicio. En este sentido, la "función fundamental de la administración implica dar caza a aquel blanco en movimiento que es el coalineamiento ..." (THOMPSON, 1967; trad. it. p. 273) entre componentes estratégicas, técnicas y estructurales.

Como conclusión, desde la óptica por procesos:

- las componentes analíticas del sistema de información son las mismas de un proceso organizativo:

- estratégicas: expresadas por los objetivos que orientan el sistema ${ }^{5}$;

- técnicas: expresadas por las acciones de transformación del input en output;

- de gestión: expresadas por las reglas de coordinación y control de las actividades y de las personas;

- los instrumentos informáticos utilizados son una de las componentes analíticas del sistema de información en términos de reglas de desarrollo de las actividades y por eso relativos a las elecciones de la gestión informativa.

\section{El proceso de desarrollo de proyectos: orientaciones estratégicas}

Nos preguntamos en este punto si es posible una caracterización general de los vínculos que las elecciones estratégicas del proceso de desarrollo de proyectos ponen a la acción de desarrollo de proyectos. Nosotros creemos que la teoría de la acción organizativa puede dar los instrumentos interpretativos para dar una respuesta afirmativa. La pregunta que nos ponemos es, en otras palabras, ¿qué grados de libertad dejan a la acción de desarrollo de proyectos las elecciones estratégicas? ¿En que medida, y hasta que punto, los objetivos del proyecto permiten un cambio del sistema de información existente?

Hacerse esta pregunta significa sobretodo, y preliminarmente, preguntarse si es posible caracterizar la variabilidad de los procesos organizativos y por lo tanto de los sistemas de información. Con esta intención resulta muy útil la esquematización propuesta por Maggi $(1990,1997)$ según la forma que está presentada en la Figura 8.

Bajo tal lógica, las componentes fundamentales del pro- 
ceso (objetivos, técnicas, modalidades de coordinación y control operativas e informativas) están ligadas por relaciones de congruencia en términos de variabilidad. Es decir, variaciones en los objetivos necesitan variaciones en las otras componentes y al revés. Esta relación no es determinista e inevitable pero es relevante allí donde se quiera mantener un adecuado nivel de congruencia entre las com- ponentes del proceso.

En este ámbito podemos asumir que:

- El desarrollo de proyectos puede estar vinculado relativamente a los flujos estratégicos del proceso, o sea no se admite variabilidad de los objetivos que caracterizan el SI;

- El desarrollo de proyectos puede estar vinculado

Figura 8: Variabilidad de los procesos organizativos.

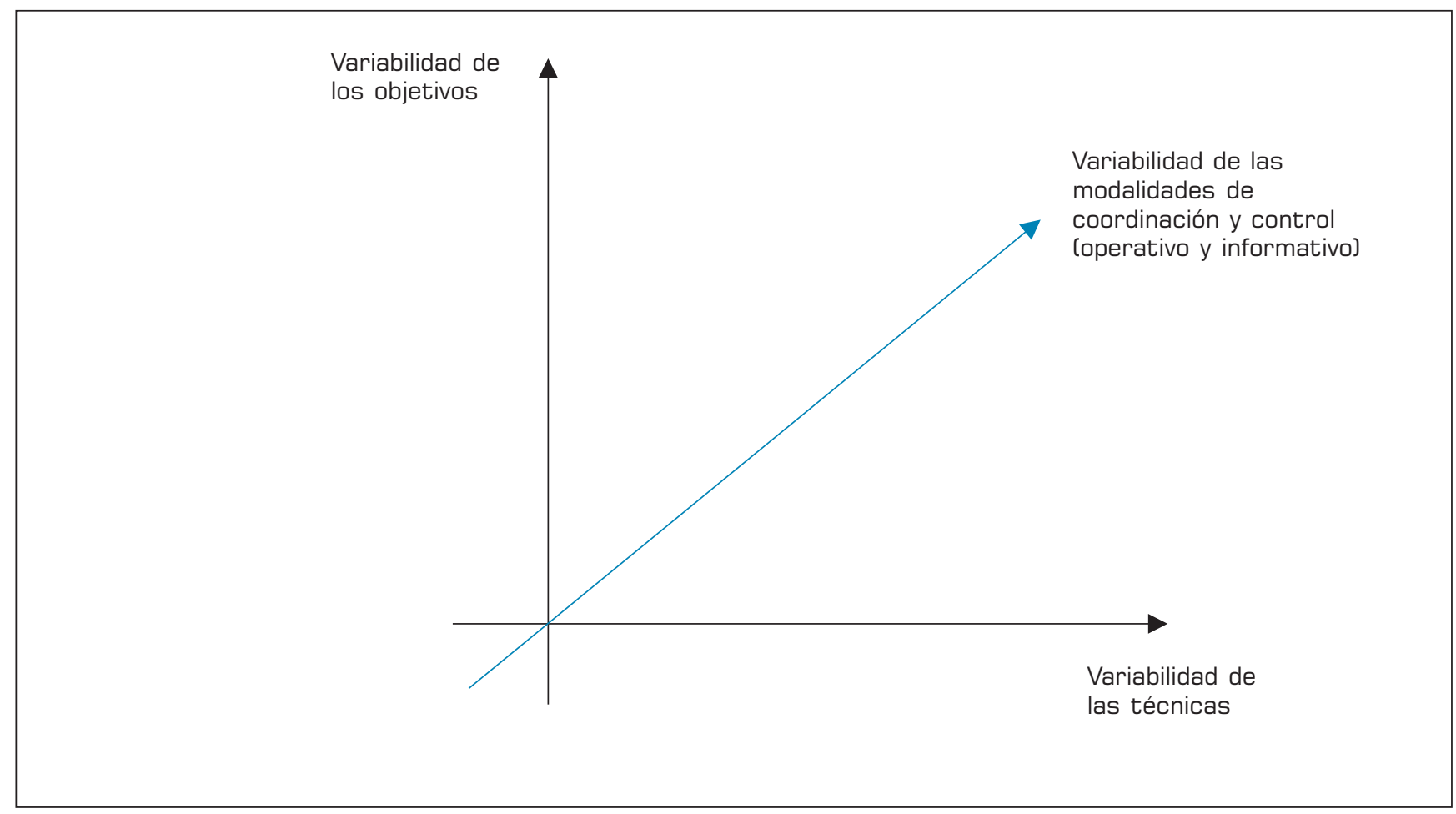

Tabla 2: Variabilidad de la técnica: profundización y ejemplos.

Es necesario en el modelo presentado, entenderse bien, una vez más, sobre el concepto de técnica. Por técnica entendemos la relación causa-efecto entre input y output, o sea que output a partir de que input y que acciones de transformación son necesarias para perseguir un determinado objetivo. Los instrumentos utilizados, en la lógica de la acción organizativa, no son elementos técnicos sino modalidades de desarrollo de las acciones técnicas y por lo tanto pertenecen a la esfera de la estructuración informativa, o sea de las modalidades de desarrollo de las actividades por parte de los actores. Seria por lo tanto fuera de la lógica del modelo pensar de interpretar, por ejemplo, el paso desde instrumentos de tipo tradicional hasta instrumentos informáticos como una variación a lo largo del eje de abcisas (técnica). El departamento de compras, una vez informatizado, seguirá transformando solicitudes de compra en ordenes de compra para cumplir su mission. No hay por lo tanto variación en los flujos técnicos mientras sí que habrá variación en los flujos de información, donde serán necesarias nuevas informaciones para el desarrollo en términos de modalidad de utilización del nuevo instrumento.

Esto podría llevar variaciones significativas también en las otras componentes del proceso. Por ejemplo, la mayor rapidez de ejecución y la automatización de las actividades más de procedimiento podrían llevar a una ampliación de la mission del departamento de compras desde la emisión de las ordenes de compra hasta la planificación de los abastecimientos (variación estratégica). En tal caso sería oportuna también una variación técnica, por ejemplo eliminando las ordenes de compra y procesando directamente las necesidades de producción. La retroacción de las elecciones de estructuración de la información (utilización de instrumentos informáticos) sobre las elecciones estratégicas y técnicas no es inevitable, la relación no es determinista pero es una oportunidad que permite elevar el nivel de congruencia del proceso. 
relativamente a los flujos técnicos donde no se admita variabilidad de las acciones de transformación de input en output (y en sus relaciones) que caracterizan el SI.

Profundizamos los dos aspectos. Examinamos la Figura 9. Esta muestra el caso del vínculo sobre la variabilidad de los flujos estratégicos que caracterizan el SI. Fijando en O la disposición estratégica, podríamos teóricamente variar la disposición técnica a lo largo de todo el eje de abcisas. De forma más realista asumimos un intervalo T1T2 de soluciones congruentes con la disposición estratégica dada. Llamamos línea de congruencia los puntos del plano $(\mathrm{O}, \mathrm{T})$ con $\mathrm{O}$ fijado y $\mathrm{T}$ comprendido entre T1 y T2. Tendremos luego naturalmente un conjunto de posibles configuraciones de los flujos de gestión congruentes con la línea de congruencia. Conceptualmente el orden de variabilidad de gestión es también representable con una línea, siendo función de una sola variable.

Suponemos ahora no admitir la variabilidad técnica y fijar la disposición técnica a la solución T. Llamamos punto de congruencia el punto del plano $(\mathrm{O}, \mathrm{T})$. En este caso la variabilidad de gestión resulta también prácticamente nula, siendo vinculada a un punto del plano.

Suponemos ahora, lo contrario, admitir la variabilidad tanto de los flujos estratégicos entre los valores $\mathrm{O} 1$ y $\mathrm{O} 2$ como de los flujos técnicos entre los valores T1 y T2.
Llamamos superficie de congruencia el cuadrado de vértices $(\mathrm{O} 1, \mathrm{~T} 1),(\mathrm{O} 2, \mathrm{~T} 1),(\mathrm{O} 1, \mathrm{~T} 2),(\mathrm{O} 2, \mathrm{~T} 2)$. Conceptualmente, en este caso la variabilidad de las soluciones de gestión se expresa por una superficie siendo función de dos variables.

En conjunto, por lo tanto, desde el punto de vista de los vínculos estratégicos, podemos distinguir entre tres orientaciones de desarrollo de proyectos:

1) Orientación a la automatización o a la innovación tecnológica: llamamos así las tipologías en las cuales tanto los objetivos como las técnicas están vinculados. En tal caso, también la variabilidad de gestión resulta fuertemente vinculada y sustancialmente limitada a los instrumentos empleados en el desarrollo de las actividades. El objetivo central del proyecto es en este caso la automatización del desarrollo de la actividad.

2) Orientación a la integración o a la innovación de gestión: responde al caso en el cual solo se admite la variabilidad de las técnicas en función de los objetivos fijados. En general, todavía, la variabilidad técnica frente a objetivos fijados es muy limitada. Asumimos por lo tanto como objetivo central de esta orientación la integración de gestión de los procesos.

3) Orientación a la innovación de proceso: en tal caso se admite variabilidad en las dos dimensiones. La acción de desarrollo de proyectos es por lo tanto orientada al

Figura 9: El proceso de desarrollo de proyectos: vínculos estratégicos.

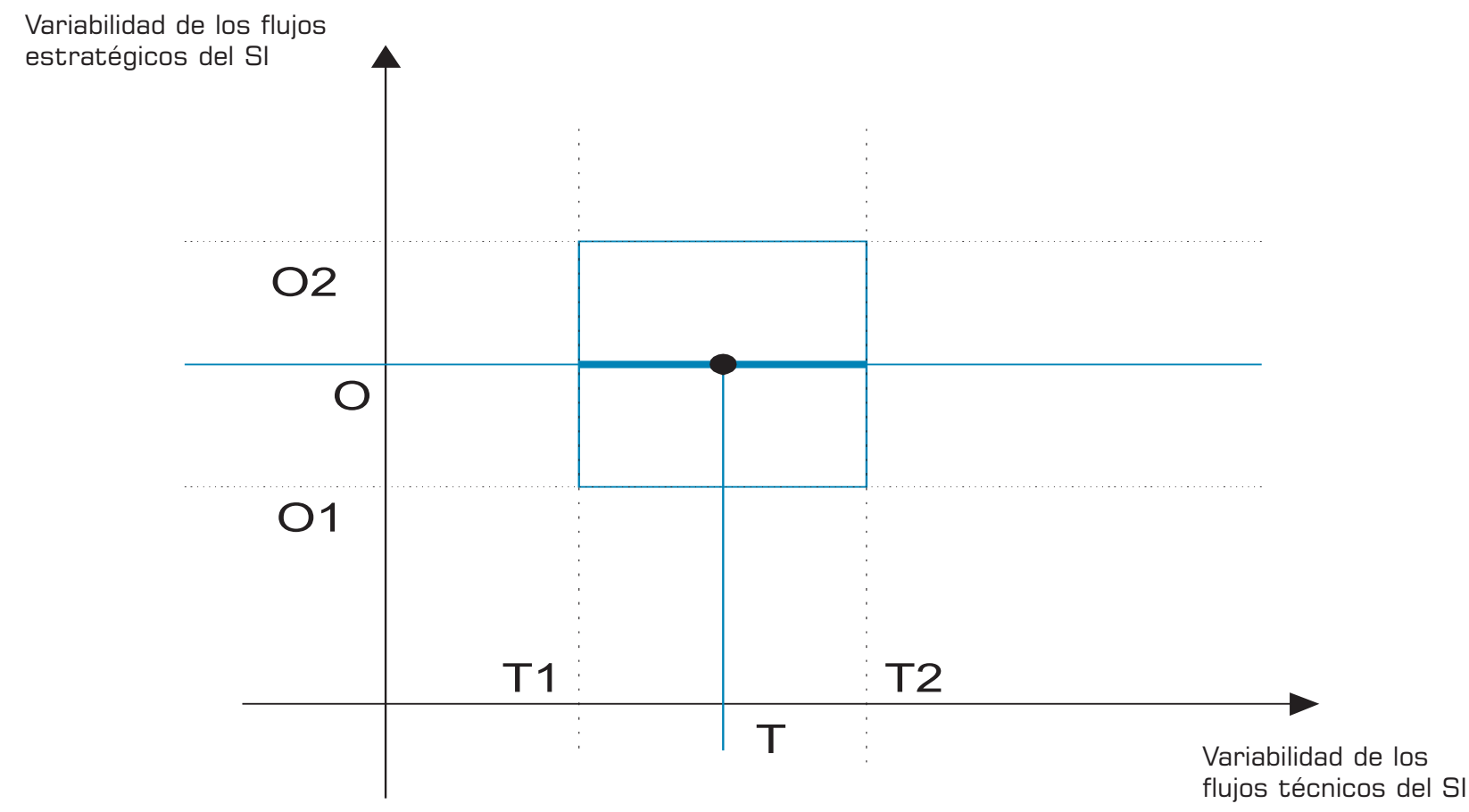


cambio de los flujos estratégicos y técnicos. En tal caso el objetivo central del proyecto es el co-alineamiento (Thompson, 1967) de las componentes estratégicas, técnicas, de gestión a través de su modificación conjunta.

Se puede notar que no indicamos una orientación sea mejor que las demás. Simplemente nos limitamos a indicar las diferentes lógicas que pueden orientar el proceso de desarrollo de proyectos ${ }^{6}$. Ellas derivan de las elecciones estratégicas de proyecto que pueden ser explícitas o implícitas con relación a las orientaciones arriba indicadas. Por ejemplo, en un caso real (RAVAGNANI, 2000a; pág. 94) la estrategia general de proyecto era explícitamente expresada en estos términos: "Tres objetivos: aumentar el grado de integración entre las diferentes sociedades del grupo a través de una homogeneización de los sistemas contables y de los procesos específicos entre compañías; para todos los otros procesos limitar al máximo el grado de innovación respecto a lo existente; entrar en producción por lo menos con las funcionalidades mínimas dentro de Octubre 1999, como manera de asegurar sin traumas el tránsito al euro en el año 2000". Una visión como esta de proyecto expresa claramente objetivos de integración entre compañías, en particular para el área contable, y de simple automatización para los otros procesos.

Finalmente es importante observar que las tres orientaciones no son alternativas sino acumulativas en el sentido que las finalidades más complejas (innovación de proceso) comprenden también aquellas menos complejas (integración de gestión y automatización).

Para aclarar lo que hemos dicho recogemos el ejemplo reelaborado por Hammer.

Hammer (1993) observó que las frecuentes discordancias entre los documentos ocupaban a los asignados a la contabilidad de proveedores cerca el $80 \%$ de su tiempo. Esto llevaba a la necesidad de un rediseño del proceso.

Confrontaremos ahora las diferentes orientaciones de desarrollo de proyectos subrayando las posibles elecciones de rediseño coherentes con cada una de ellas.

1) Orientación a la innovación tecnológica: en este caso se asumen como datos tanto los objetivos, o sea las mission de los procesos, como las técnicas, o sea las acciones de transformación, los input y los output, y el proceso, en términos de ordenación de las actividades técnicas. La intervención, dados los vínculos indicados, se referirá por lo tanto primariamente a los instrumentos empleados para el desarrollo (Figura 10). Se podrán obtener además modificaciones en el formato de algunos input y output que podrán ser informatizadas a través de bases de datos. En este caso podrían cambiar también algunas reglas de gestión operativa, por ejemplo ya no será necesario que el departamento de compras produzca y transmita una copia de la orden de compra. La individuación de incongruencias en los documentos será probablemente más rápida, pero la necesidad de intervenciones de adaptación mutua entre las unidades para hacer frente a las incongruencias continuará.

2) Orientación a la innovación de gestión: en este caso, además de las intervenciones arriba indicadas, hay otros márgenes de maniobra en términos de variabilidad técnica pero no de objetivos (Figura 11). Por ejemplo, los ordenes de compra podrían ser visibles también desde el almacén que podría así de una manera inmediata, eventualmente con una señalización automática, evidenciar incongruencias entre lo que se ha recibido y la orden de compra. Esto requiere también una intervención de gestión. En el caso que se examina, la regla de conexión entre recepción y combinación de documentos incluye también la verificación de congruencia entre AEM y OC. En términos de flujos de información, está expresado el hecho que el almacén activará comunicaciones con la contabilidad donde encuentre incongruencias entre los dos documentos. Entonces la contabilidad de proveedores se activará para individuar las causas, a través de procesos de comunicación con compras y proveedores, comunicando luego las acciones a emprender al almacén.

3) Orientación a la innovación de proceso: en este caso, la intervención se refiere también a una redefinición de la mission de los procesos. En particular, Hammer (1993) reposicionó la contabilidad de proveedores sobre un único objetivo de pago al proveedor por los productos recibidos (Figura 12). El emparejamiento entre productos recibidos y OC fue encargado al almacén, que podía disponer de toda la información necesaria (Figura 13). Una vez efectuado el emparejamiento, por parte del almacén, el departamento de compras procedería al pago sin esperar la factura y, por lo tanto, demandando al proveedor la emisión de una factura congruente con cuanto ha sido pagado. Es evidente la simplificación de los flujos de gestión operativa y, sobretodo de gestión informativa que no requieren más, sino en mínima medida, intervenciones de ajuste mutuo.

\section{El proceso de desarrollo de proyectos: orientaciones por proyectos}

Hemos visto en el párrafo anterior como el proceso de desarrollo de proyectos puede ser vinculado estratégicamente. Es decir, como los objetivos asignados al proceso pueden condicionar la lógica de las opciones de desarrollo de proyectos según diferentes orientaciones. 
Figura 10: Rediseño del proceso orientado a la automatización.

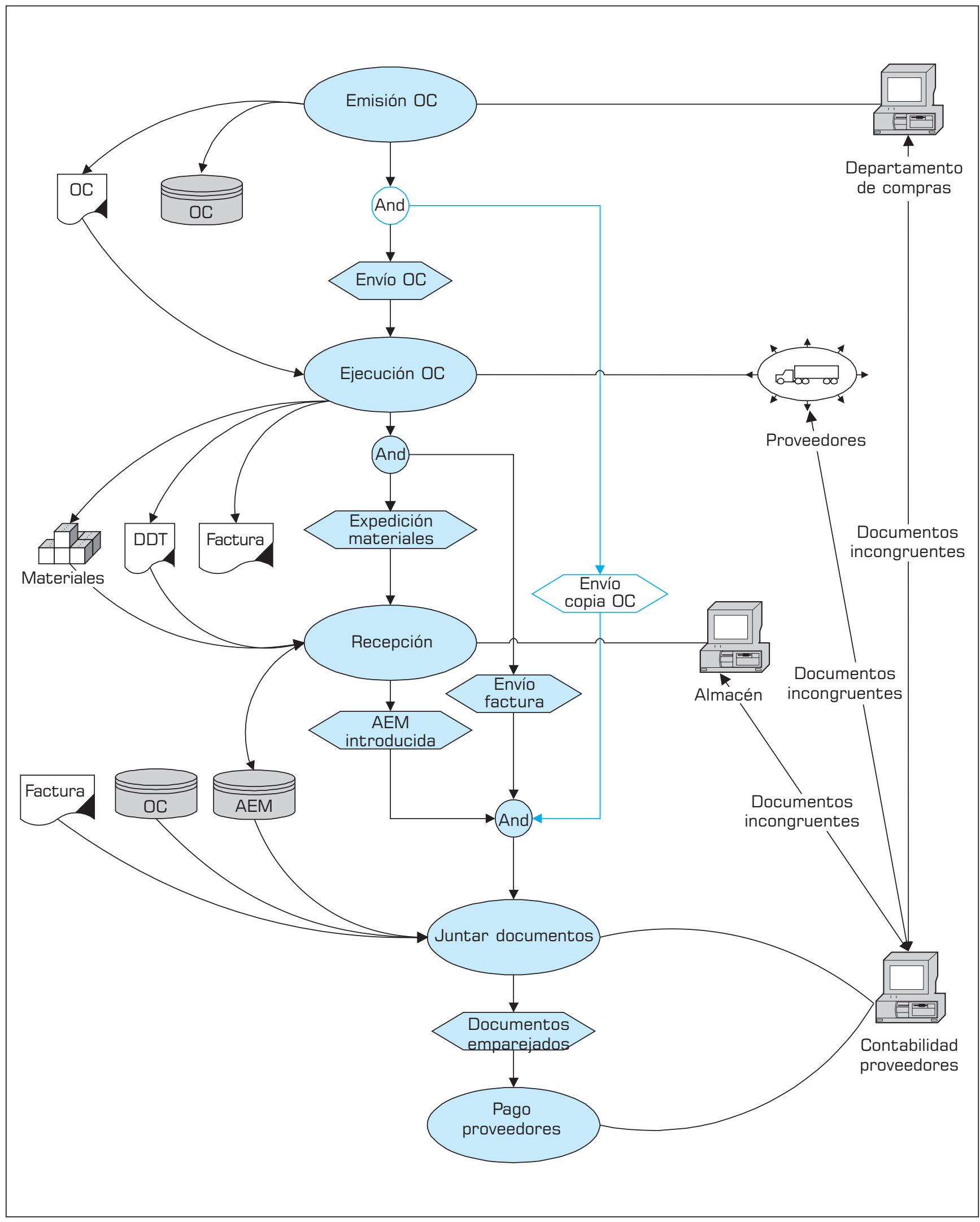


Figura 11: Rediseño de un proceso orientado a la integración de la gestión.

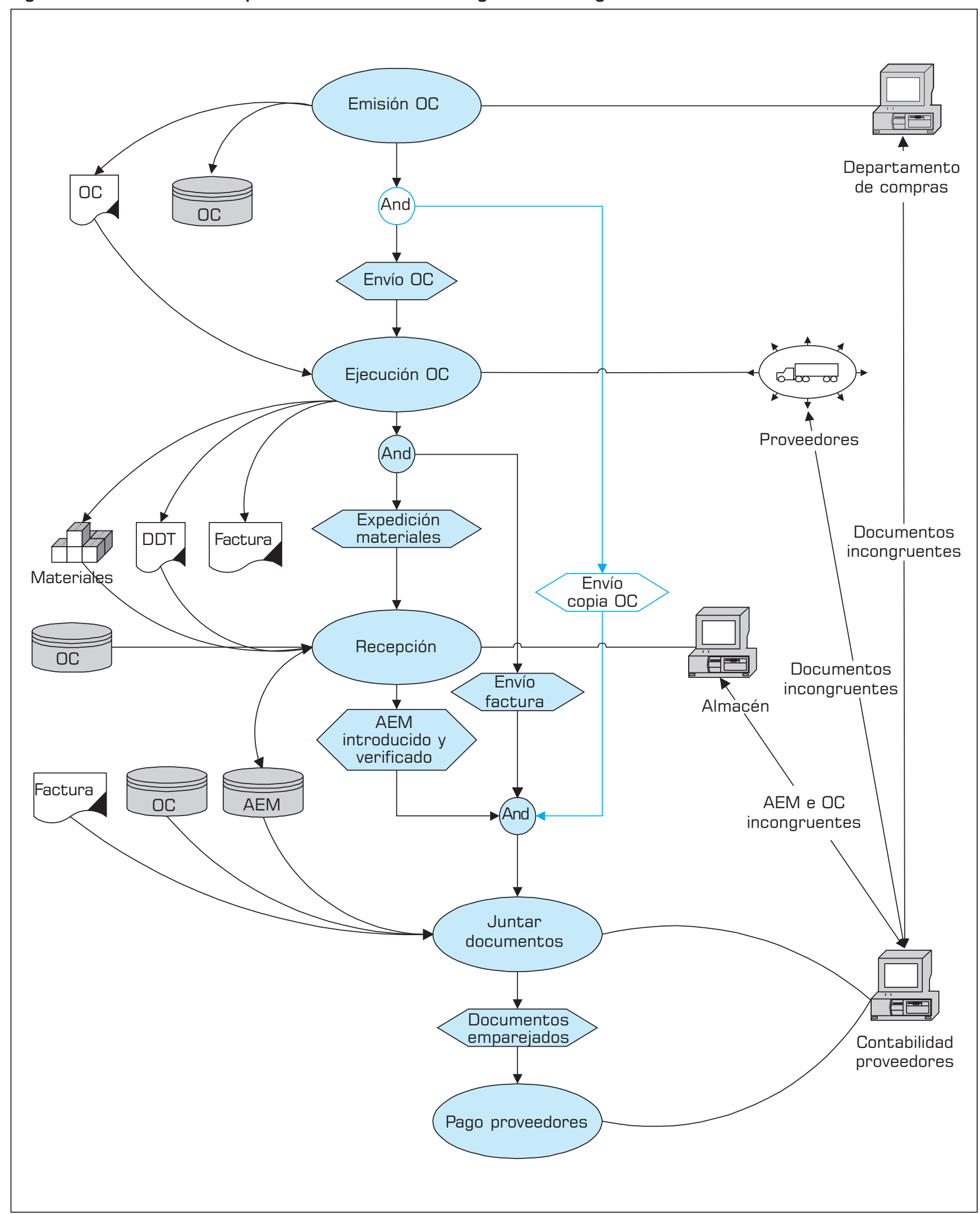


En este párrafo queremos en cambio desarrollar algunas consideraciones a cerca de las orientaciones por proyectos que puedan guiar el desarrollo de proyectos. Esto requiere una profundización de las lógicas que caracterizan las opciones técnicas del proyecto. Hacemos referencia a la figura 1. Aquí hemos representado como opciones técnicas el conjunto de decisiones relativas a la transformación del anterior SI en el nuevo SI. Estas pueden ser divididas en (MASINO, ZAMARIAN, 2000) (figura 14):

- opciones de adopción: definen los requerimientos del nuevo sistema en términos de flujos estratégicos, de transformación y de gestión; en otros términos el output de esta fase es denominado to be model, o sea la indicación, más o menos explícita, de cómo deberá ser el nuevo proceso;

- opciones de configuración: expresan las opciones de configuración del software que será implementado con relación a los requerimientos expresados en la fase anterior; el output en esta fase es un prototipo, o sea un paquete de software configurado sobre la base de determinados requerimientos pero aún no ratificado y hecho operativo para la utilización concreta;

\section{Figura 12: Contabilidad de proveedores: rediseño de los flujos estratégicos.}

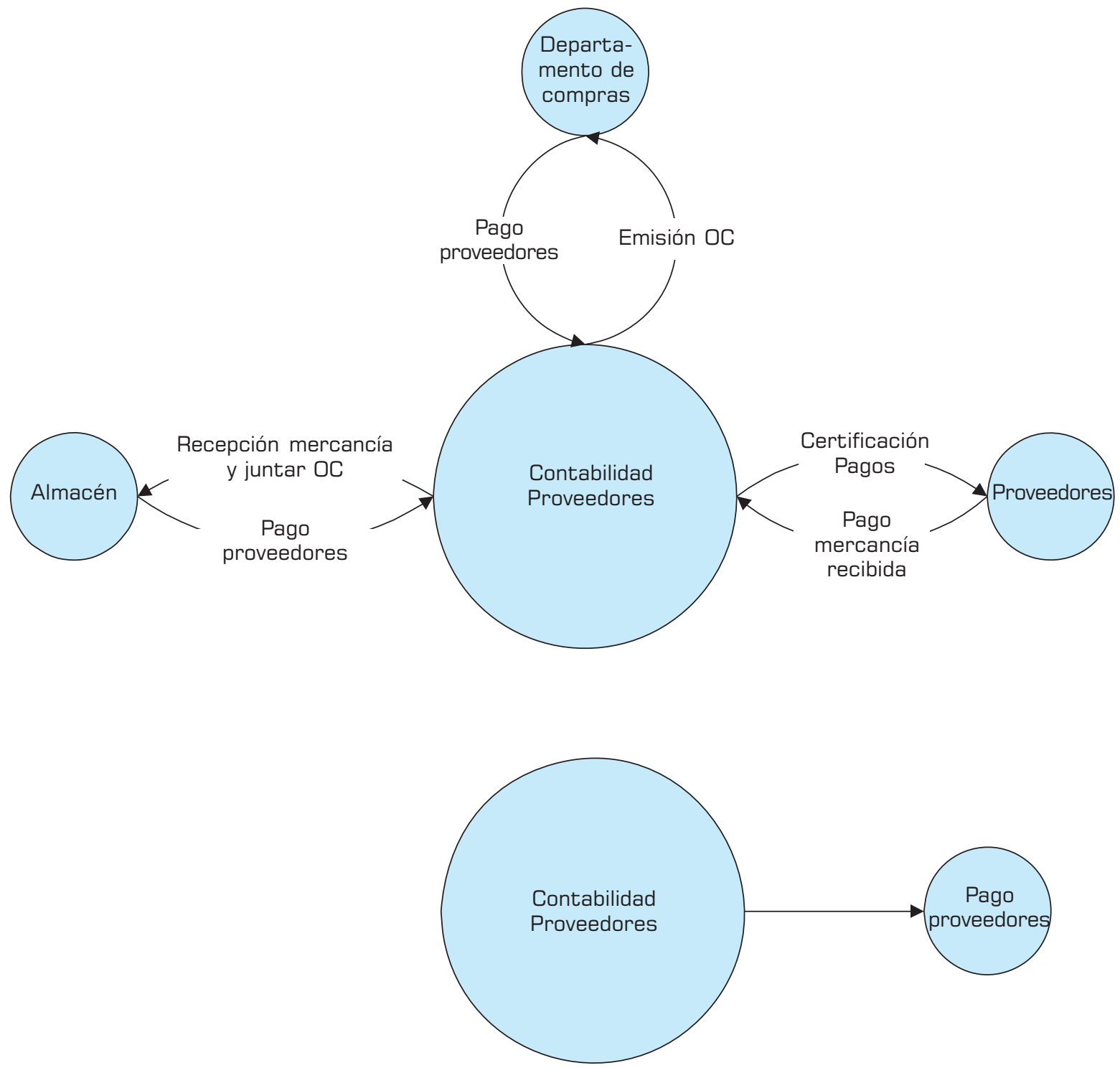


- opciones de utilización: expresan las modalidades con las cuales el prototipo será efectivamente utilizado por los usuarios finales. En este sentido, las opciones de utilización "pueden ser también bastante divergentes respecto a las opciones de adopción y de desarrollo de proyectos, aunque estas sean necesariamente, por lo menos en parte influenciadas." (MASINO, ZAMARIAN, 2000; pág. 155). En estos términos, por lo tanto, solo en esta fase podemos hablar de "nuevo sistema de información".

Podemos, llegados a este punto, distinguir diferentes enfoques técnicos al desarrollo de proyectos en relación con la manera de entender las relaciones entre las diferentes fases decisionales.

Figura 13: Rediseño orientado a la innovación de proceso.

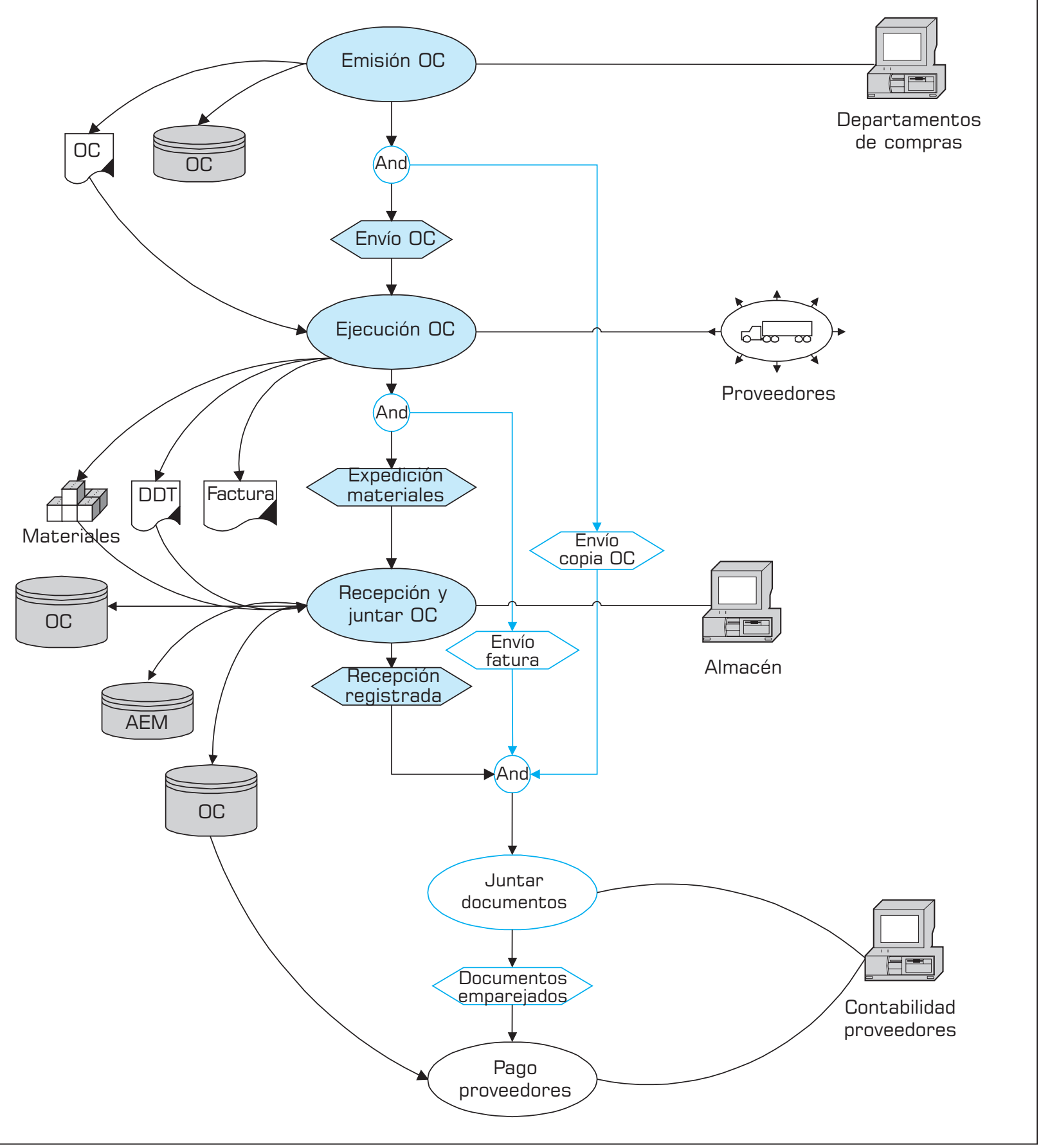


En este sentido hablaremos de:

- incertidumbre sobre las opciones de adopción: si hay incertidumbre sobre las opciones de adopción cuando el nivel de cambio requerido es elevado y la complejidad de los procesos alta. En tal caso el to be model no puede entenderse como un input dado e inmodificable sino que requerirá continuos ajustes con relación a las opciones de configuración y de utilización;

- incertidumbre sobre las opciones de utilización: si hay incertidumbre sobre las opciones de utilización en caso de procesos escasamente formalizados y estructurados en los cuales los actores gozan de elevada discrecionalidad. En este caso las opciones de utilización implican frecuentes retroacciones tanto sobre las opciones de configuración como sobre las de adopción.

Ahora podemos caracterizar en términos dicotómicos (alto, bajo) el nivel de incertidumbre que caracteriza las dos dimensiones, obteniendo cuatro orientaciones de desarrollo de proyectos (Tabla 3).

1. Orientación informática: denominamos así las situaciones en las cuales se asume la certeza tanto en las opciones de adopción como en las de utilización. La expresión típica de esta lógica es el mencionado modelo del ciclo de vida en el cual los momentos de definición de requerimientos, diseño del sistema e implementación

Figura 14: El desarrollo de proyectos: opciones técnicas.

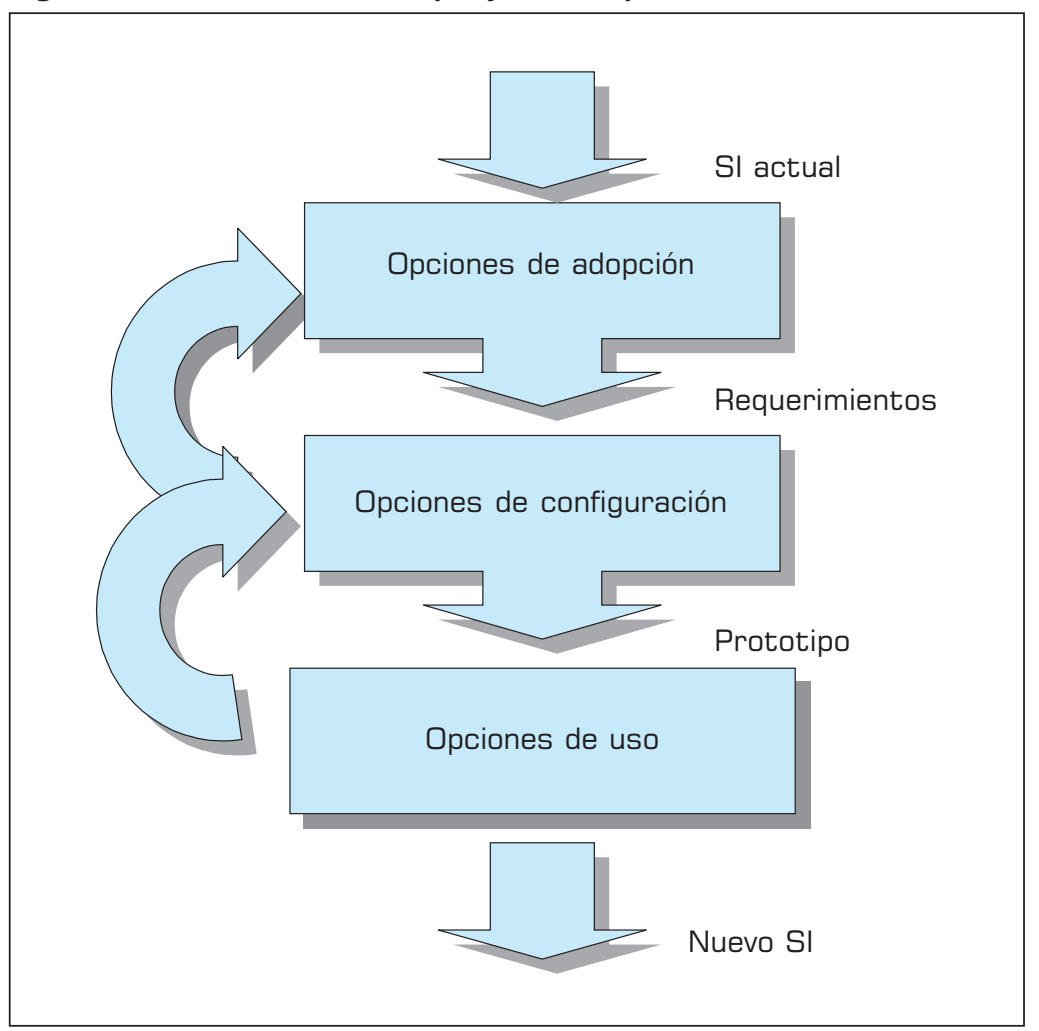

están rígidamente separados y secuencializados. En tal caso el centro del proyecto está en las opciones de configuración.

2. Orientación funcional: en tal caso se asumen como dados los requerimientos mientras que se asumen como inciertas las opciones de utilización. Respetan esta lógica tanto los enfoques de tipo prototipaje como aquellos con raíz socio técnica. El centro del desarrollo de proyectos, desde esta óptica, está en la configuración, en la utilización y en las retroacciones entre los dos momentos.

3. Orientación a la vision: desde esta óptica, simétrica a la anterior se asumen como principales fuentes de incertidumbre las opciones de adopción. Las opciones de configuración son consideradas como una palanca importante de rediseño de los procesos, razón por la cual la relación entre los dos momentos decisionales (adopción y configuración) no puede que ser de tipo recíproco. Respetan esta lógica muchas aproximaciones orientadas al BPR. En este caso el punto de apoyo del desarrollo de proyectos es la definición de la visión del nuevo proceso. Una vez definida, en términos de iteraciones entre elecciones de adopción y configuración, las opciones de utilización son consideradas escasamente problemáticas.

4. Orientación por procesos: en tal caso se asume la incertidumbre sobre ambas dimensiones. Se asumen por lo tanto las opciones de configuración relevantes a las finalidades del diseño de los nuevos procesos, y caracterizadas entonces por relaciones recíprocas con las opciones de adopción. De la misma manera, se reconoce que el proyecto del proceso y su desarrollo concreto, expresado por las opciones de utilización, no pueden ser separados, siendo caracterizados por momentos relevantes de retroacción.

\section{Congruencia entre orientaciones estratégicas y orientaciones por proyectos}

En este párrafo trataremos algunas conclusiones relacionadas con las consideraciones desarrolladas hasta ahora.

El lector ya habrá notado que en los párrafos precedentes nos hemos limitado a describir las diferentes orientaciones, tanto estratégicas como por proyectos, sin, todavía, haber expresado juicios de valor sobre las diversas lógicas presentadas. Se desilusionará quien espere encontrarlos en este párrafo. De hecho, pensamos que no existen en absoluto enfoques mejores que otros, sino que un enfoque es más adecuado que el otro en función de la congruencia entre orientaciones estratégicas y por proyectos. Así, por ejemplo, el 
clásico modelo del ciclo de vida ha dado resultados positivos en el caso de proyectos relacionados con procesos poco complejos y orientados a la automatización. Al contrario, proyectos complejos y fuertemente orientados a la innovación de procesos encuentran en el modelo del ciclo de vida y, más en general, en los instrumentos típicos de la software engineering más vínculos que recursos utilizables (DAVENPORT, 1994).

El problema puede ser resumido como en la tabla 4. Las situaciones de congruencia se muestran en las casillas ralladas de la diagonal principal ${ }^{7}$.

Sobre la diagonal principal tenemos las opciones ineficaces. La orientación informática, como ya hemos dicho, se concilia mal con orientaciones estratégicas más complejas que la simple automatización. Debajo de la diagonal principal tenemos en cambio, situaciones ineficientes debidas a que el conjunto de las competencias y la complejidad metodológica de las orientaciones por procesos pueden ser desproporcionadas respecto a los objetivos de simple innovación de los instrumentos técnicos empleados.

De todos modos, se observa, a modo de conclusión, que raramente la orientación estratégica del proyecto está claramente definida y compartida. En tal caso, el enfoque por procesos resulta el único punto de partida racional respecto a las premisas del proyecto allí donde permite la incorporación en el proyecto de las opciones adoptadas.

\section{Conclusiones y puntos abiertos}

El trabajo presentado nos ha permitido definir las opciones epistemológicas sobre las cuales, a nuestro parecer, debe fundamentarse una metodología de desarrollo de proyectos de los sistemas de información. En particular han sido definidos, dentro de un único marco de referencia teórico, los conceptos de desarrollo de proyectos de sistemas de información y del sistema de información.

Basándonos en ellos hemos individualizado las posibles orientaciones estratégicas que caracterizan el desarrollo de proyectos. Respecto a este punto remarcamos que no hemos argumentado sobre la racionalidad de las posibles estrategias de proyecto, asumiéndolas, en cambio, como un dato de partida. Una discusión sobre la racionalidad intrínseca de las posibles estrategias de proyecto se sale de los objetivos de este trabajo. Hemos individualizado las lógicas de congruencia entre orientaciones estratégicas y orientaciones por proyectos en términos de relaciones entre opciones de adopción, de configuración y de utilización.

Para una caracterización completa de las orientaciones por proyectos deberían ser afrontados algunos puntos que aquí no han sido tratados y que son indispensables para el desarrollo de una metodología completa de desarrollo de proyectos aunque son, lógicamente, secundarios respecto a las consideraciones aquí tratadas. En particular, pretendemos, en trabajos futuros, completar el tratamiento relativo a los siguientes temas:

- Lógica por proyecto e implicación de los usuarios: ¿quienes son los usuarios? ¿Cómo pueden ser considerados con relación a las decisiones que deben ser introducidas en el desarrollo de proyectos? ¿En qué términos y cuando deben ser implicados? ¿Según qué modalidad con relación a las diferentes orientaciones por proyectos?

- Complejidad del proyecto y gestión del proyecto: ¿Cuáles son las posibles opciones relacionadas con la

Tabla 3: Orientaciones por proyectos.

\begin{tabular}{|l|l|l|}
\hline & \multicolumn{2}{|c|}{ Incertidumbre sobre las opciones de adopción } \\
\hline $\begin{array}{l}\text { Incertidumbre sobre las } \\
\text { opciones de utilización }\end{array}$ & Baja & Alta \\
\hline Baja & Orientación informática & Orientación a la visión \\
\hline Alta & Orientación funcional & Orientación por procesos \\
\hline
\end{tabular}

Tabla 4: Congruencia entre orientaciones estratégicas y orientaciones técnicas.

\begin{tabular}{|l|l|l|l|}
\hline & \multicolumn{3}{|c|}{ Orientaciones estratégicas } \\
\hline $\begin{array}{l}\text { Orientaciones } \\
\text { por proyectos }\end{array}$ & $\begin{array}{l}\text { Orientación a la } \\
\text { innovación tecnológica }\end{array}$ & $\begin{array}{l}\text { Orientación a la } \\
\text { innovación de gestión }\end{array}$ & $\begin{array}{l}\text { Orientación a la } \\
\text { innovación del proceso }\end{array}$ \\
\hline Orientación informática & & & \\
\hline Orientación funcional & & & \\
\hline Orientación por proceso & & & \\
\hline
\end{tabular}


gestión, es decir en la coordinación y control del proyecto? ¿Cómo se diferencian con relación a la complejidad del proyecto, es decir a las orientaciones estratégicas y por proyectos que lo caracterizan?

- Valoración del proyecto y valoración del sistema: ¿Cuáles son los posibles criterios de valoración de las acciones por proyecto y del output del desarrollo de proyectos, es decir del sistema de información? ¿Qué relaciones hay entre valoración del desarrollo de proyectos y la valoración del sistema proyectado? ¿Quiénes son los que valoran y quienes los que son valorados?

Como se puede observar, se trata de cuestiones relevantes para construir un aparato teórico completo y conceptual sobre el cual basar una metodología de desarrollo de proyectos. Sobre estas preguntas pretendemos orientar nuestra investigación en un futuro próximo.

\section{- Notas}

${ }^{1}$ La compatibilidad epistemológica entre la Structuration Theory de Giddens y la Teoría de la Acción Organizativa ha sido sostenida por Masino (1997). El acercamiento con las orientaciones BPR requeriría alguna precisión ulterior que se alejaría demasiado de las finalidades del trabajo. Nos limitamos a observar que el límite principal del BPR parece ser la debilidad interpretativa de los modelos que lo orientan. Sobre el plano epistemológico, todavía, el concepto de business process es bastante próximo al concepto de proceso propio de la teoría de la acción organizativa.

${ }^{2}$ Maggi (1990) habla de "proyectos de acciones y decisiones". Nosotros utilizaremos el término flujo entendido como flujo de acciones y decisiones.
${ }^{3}$ La solución indicada por Hammer es de hecho entendida como una Best Practice incorporada en todos los sistemas ERP.

${ }^{4}$ Thompson (1967) distingue tres tipologías tecnológicas con relación a las diversas problemáticas que pone respecto a su valoración en términos de racionalidad técnica:

1) Tecnología de concatenación. Se trata de procesos de transformación caracterizados por secuencias estables y bien definidas de fases. Su característica es la repetitividad que los hace fácilmente aproximables a las condiciones de perfección técnica.

2) Tecnología intensiva. Su especificidad es debida a la no repetitividad de la secuencia y combinaciones de las diversas fases que dependen del feedback proveniente del objeto de la transformación. En general, los procesos caracterizados de esta forma, pueden ser valorados en términos de eficacia pero no de eficiencia. Es decir que no es posible saber cual es el nivel óptimo del empleo de los recursos para obtener un dato resultado. Organizaciones típicamente caracterizadas por tecnología intensiva son las hospitalarias. 3) Tecnología de mediación. Caracteriza las organizaciones cuya finalidad principal concierne la conexión de usuarios distribuidos en el tiempo y en el espacio que intentan ser interdependientes. La problemática principal de esta variedad tecnológica concierne la clasificación y la estandarización de las modalidades de las interacciones con los usuarios. Ejemplos típicos son los bancos, las sociedades de telecomunicaciones, etc.
${ }^{5}$ Los términos sistema y proceso son utilizados como sinónimos siempre y cuando el sistema esté dotado de la propiedad de multifuncionalidad, es decir capacidad de cambiar los propios objetivos, y morfogénesi, es decir capacidad de cambiar las propias estructuras. Maggi, 1990)

${ }^{6}$ Nos limitamos aquí a observar que la mayoría de la literatura del último decenio opina que los beneficios obtenibles sean tanto más altos cuanto más altos son los grados de libertad admitidos en términos de modificaciones estratégicas, técnicas y de gestión.

${ }^{7}$ Por razones de simplicidad hemos unido orientación a la vision y orientación por proceso.

\section{- Bibliografia}

BARLEY, S.R. 1986. Technology as an occasion for structuring: evidence from observations of CT scanners and the social order of radiology departments. Administrattive Science Quarterly. Vol. 31 n.1, pp. 78-108.

BARLEY S.R. 1990. The alignement of technology and structure through roles and networks. Administrative Science Quarterly. Vol. 35, n.1, pp. 61-103.

BARNARD C. 1938. The Functions of the executive. Cambridge (Mass.). Harvard University Press. (trad. it. Le funzioni del dirigente, Utet, Turín, 1970)

BRACCHI, G. MOTTA, G. 1998. Processi aziendali e sistemi informativi. Milán. Angeli.

COOMBS, R. HULL, R. 1997. The wide research context of Business Process Analysis. Manchester. CROMTEC.

DAVENPORT, T.H. 1994. Innovazione de processi. Milán. Angeli.
DE SANCTIS, G.; POOLE, M.S. 1994 Capturing the complexity in advanced technology use : adaptive structuration theory. Organization Science. Vol. 5, n.2, pp. 121-147.

HALL, H. 1977. Organizations, Structure and Process. Englewood. Prentice-Hall.

HAMMER, M.; CHAMPY, J. 1993. Reengineering the corporation. Nueva York. Harper Business.

MAGGI, B. 1990. Razionalità e benessere. Studio interdisciplinare dell'organizzazione. Milán, Etas.

MAGGI, B. ALBANO, R. 1997. La teoria dell'azione organizzativa. In Costa, G. Nacamulli, R.C.D. (a cargo de) Manuale di organizzazione aziendale. Vol. 1.:220249. Turín. UTET.

MASINO, G. 1997. Nuove regole di progettazione. Roma. La Nuova Italia Scientifica.

MASINO, G. 2000. Nuove tecnologie e azione organizzativa.Turín. ISEDI.
MASINO, G.; ZAMARIAN, M. 2000. La mediazione degli artefatti nella regolazione organizzativa. In Maggi, B. (a cargo de) Le sfide organizzative di fine e inizio secolo. Milán. ETAS.

MIGLIARESE, P.; FERIOLI, C. 1996. Supporting organisational relations through information technology in the innovative organisational forms. European Journal of Information Systems, 5: 196:207.

MIGLIARESE, P. FERIOLI, C.; IAZZOLINO; G. 1999. Dimensione organizzativa del Business Process Reengineering: la necessità di un chiarimento. Studi Organizzativi, 2: 55-81.

ORLIKOWSKI, W. BAROUDI, J.J. 1991 Studying IT in organization: research approaches and assumptions. Information Systems Research. 2(1), pp. 1-28.

ORLIKOWSKI, W.J. 1992. The duality of technology: rethinking the concept of technology in organizations. Organization Science. Vol. 3, n. 3, Agosto, pp. 398-427.
PACE, R.W. 1983. Organizational Communication. Englewood. Prentice-Hall.

PEPPARD, J. 1994. Where is BPR going? Paper presentato alla conferenza dell'UNICOM "Improving Business Performance Through Effective Use of IT". Londres. 7-9 Junio.

PONTIGGIA, A. 1997. Organizzazione de Sistemi Informativi. Milán. Etas Libri.

RAVAGNANI, R. 2000a. Patologie Organizzative Associate ai Sistemi Informativi Integrati. Economia \& Management, 3: 85-98.

RAVAGNANI, R. 2000b. Information Technology e gestione del cambiamento organizzativo. Milán. Egea.

SIMON, H.A. 1947. Administrative Behavior. Nueva York. Mcmillan.

THOMPSON, J.D. 1967. Organization in action. Nueva York. McGraw-Hill.

TYRE, J.; ORLIKOWSKI, W.J. 1994 Windows of opportunity: temporal patterns of technological adaptation in organizations. Organization Science. Vol.5, n.1, Febrero, pp. 98-118. 